Horizons philosophiques

\title{
Une notable lettre anonyme dans la Querelle des Bouffons
}

\section{Akiko Koana}

Volume 13, numéro 2, printemps 2003

Herder (1744-1803) : le clair-obscur

URI : https://id.erudit.org/iderudit/801234ar

DOI : https://doi.org/10.7202/801234ar

Aller au sommaire du numéro

\section{Éditeur(s)}

Collège Édouard-Montpetit

\section{ISSN}

1181-9227 (imprimé)

1920-2954 (numérique)

Découvrir la revue

Citer cet article

Koana, A. (2003). Une notable lettre anonyme dans la Querelle des Bouffons. Horizons philosophiques, 13(2), 33-42. https://doi.org/10.7202/801234ar d'utilisation que vous pouvez consulter en ligne.

https://apropos.erudit.org/fr/usagers/politique-dutilisation/ 


\section{UNE NOTABLE LETTRE ANONYME \\ DANS LA QUERELLE DES BOUFFONS}

L'histoire de l'esthétique musicale au XVIIIe siècle en France se caractérise par les querelles continuelles entre la musique française et la musique italienne. Elle fut marquée par trois grandes querelles. La première est celle entre l'abbé Raguenet ${ }^{1}$ et le Cerf de la Viéville'2. La deuxième est celle dite "des Bouffons". La troisième est celle des Gluckistes et des Piccinnistes ${ }^{3}$. Certes, ces trois querelles ont vu le jour dans des circonstances différentes et leur sujet était tout autre. Mais il ne faut pas oublier que l'adversaire (la musique italienne) était toujours le même et que ces querelles ont toutes contribué à la formation de la notion de style national4.

II faut noter aussi que l'on peut voir se développer peu à peu la nouvelle tendance de la théorie musicale qui essaie de surmonter la particularité de style national et de théoriser sur l'aspect universel du langage musical. On peut dire que cette série de disputes a justement fourni le lieu d'interaction entre ces deux types de théorie musicale qui se nourrissaient en s'opposant. À l'occasion de notre table ronde sur le thème de «l'universel et le particulier dans l'esthétique musicale au XVIIIle siècle», mon propos s'efforce de le démontrer en prenant l'exemple sur «la Querelle des Bouffons».

\section{La Querelle des Bouffons}

Parmi ces trois querelles mentionnées plus haut, la présente étude se concentre sur la deuxième querelle, c'est-à-dire, "la Querelle des Bouffons" qui est la plus acharnée des trois et qui a impliqué beaucoup de philosophes comme Diderot, d'Alembert et J.-J. Rousseau. Le mot "bouffon" dérive de celui italien buffo qui signifie "acteur comique». Plus précisément, il signifie un acteur de l'opera buffa qui est un genre de l'opéra comique italien créé à Naples au début du $18^{\theta}$ siècle comme intermède de l'opera seria. J.-B. Pergolesi est l'un des compositeurs les plus célèbres dans ce genre.

En août 1752, une troupe de l'opera buffa vint de Naples à Paris et joua Serva padrona de Pergolesi. L'accueil enthousiaste du public parisien vexa certains critiques et provoqua des disputes. C'est 
pourquoi nous appelons ces disputes "querelle des Bouffons". II faut, toutefois, mentionner au moins deux autres raisons pour expliquer l'apparition de cette querelle acharnée dans la France de cette époque.

Premièrement, le milieu du 18 ème siècle est un passage décisif de l'époque baroque à l'époque classique. Même le public français attendait quelque chose de nouveau et cependant l'Opéra de Paris continuait de présenter presque le même répertoire depuis la mort de J.-B Lully. Par exemple, la troisième reprise ${ }^{5}$ de l'opéra Omphale de Destouches en 1752 montre clairement cette situation. On pourrait mentionner à ce propos le fait que ce fut justement cette troisième reprise qui occasionna La Lettre sur Omphale de Grimm, pamphlet qui commença "la Querelle des Bouffons" au sens large du terme, comme on en parlera plus bas. La majorité du public de l'Opéra s'ennuya.

Deuxièmement, cette époque coïncide avec celle de la révolution sociale en France. Certes, nous devrons attendre la révolution politique française jusqu'en 1789 , mais toutes les préparations avaient déjà été avancées au milieu de ce siècle. Le premier volume, par exemple, de l'Encyclopédie apparut en 1751 et les principaux participants de la querelle furent, comme je l'ai déjà mentionné plus haut, des Encyclopédistes. Pour éclaircir le deuxième point, il faut parler de la signification de la musique, ou plus précisément, de l'opéra à cette époque. II ne faut pas oublier que l'opéra français fut établi avec la fondation de l'Académie Royale de Musique en 1672. À cette époque l'opéra fut un des événements organisés par l'État. Dans ce sens on peut dire que l'opéra était la nation et la nation était l'opéra. Par exemple, critiquer l'opéra de Lully, cela signifiait critiquer la monarchie absolue de Louis XIV. Par conséquent, on ne devrait pas négliger le fait que la Querelle des Bouffons ait été à la fois une dispute musicale et politique.

Quant aux documents, nous avons trois volumes de La Querelle des Bouffons édités par Denise Launay6. Ils contiennent 61 documents de 1751 à 1754 . Nous pouvons diviser ces documents en trois parties ${ }^{7}$. La première partie commence par la «Lettre sur Omphale»8 de Grimm et finit par la "Lettre à Grimm»9 de J.-J. Rousseau. Précisément parlant, cette partie n'appartient pas à la Querelle des Bouffons au sens strict du terme, parce que ces documents datent d'avant l'arrivée des «Bouffons». Elle peut être considérée plutôt 
comme le prélude du conflit principal qui naquit après la représentation de Serva padrona à Paris. Même si elle n'est qu'une escarmouche, elle montre que le fruit était mûr avant la tournée des "Bouffons". La représentation de Serva padrona jeta seulement de l'huile sur le feu.

La seconde et la troisième partie couvrent "la Querelle des Bouffons" proprement dite. Ce qui a détourné le cours de la querelle, ce fut "la Lettre sur la Musique Françoise» de J.J. Rousseau. Approximativement parlant, dans la seconde partie on parle des Bouffons et dans la troisième partie on discute de la "Lettre" de Rousseau.

\section{"Lettre sur la Musique Françoise» de J.-J. Rousseau}

La troisième partie commence, donc, par la «Lettre sur la Musique Françoise" de J.J. Rousseau. Dans cette troisième partie, on peut dire qu'il joue le rôle principal dans la querelle. Presque tous les documents écrits après sa "Lettre sur la Musique Françoise" expriment un profond ressentiment envers lui. Ou plus précisément, ils sont écrits pour le réfuter. La quantité de documents rédigés contre lui montre combien cette "Lettre» a humilié le monde parisien.

Pourquoi sa "Lettre» leur a-t-elle causé une telle contrariété? Nous pouvons indiquer deux raisons. L'une concerne son contenu. Sa négation de l'existence de la musique française n'a pas été acceptable pour les Français. Elle les a insultés et elle a attaqué leur nationalisme. L'autre concerne son style. Le dernier paragraphe de sa "Lettre» est surtout très agressif. En lisant les documents qui ont paru après sa "Lettre", on peut comprendre combien ce paragraphe a choqué les auteurs des pamphlets parce que presque tous les auteurs citent une partie de ce paragraphe pour le réfuter. Pour démontrer ces points, nous citons ce dernier paragraphe :

Je crois avoir fait voir qu'il n'y a ni mesure ni mélodie dans la Musique Françoise, parce que la langue n'en est pas susceptible; que le chant François n'est qu'un aboiement continuel, insupportable à toute oreille non prévenue; que l'harmonie en est brute, sans expression, et sentant uniquement son remplissage ${ }^{11}$ d'Écolier; que les airs François ne sont point des airs; que le récitatif François n'est point du récitatif. D'où je conclus que les François n'ont point de Musique et n'en peuvent avoir, ou que si jamais ils en ont une, ce sera tant pis pour eux ${ }^{12}$. 
De ce paragraphe il y a un commentaire raisonnable de d'Alembert. Selon lui, Rousseau aurait dû écrire "ce que nous appelons de la Musique \& qui n'en est pas" "au lieu d'employer le terme de Musique Françoise» 13 . Bien sûr, si Rousseau avait rédigé sa "Lettre" de cette façon, son message aurait été plus logique et moins agressif, donc le puissant effet de ce paragraphe se serait dissipé.

Quel est cet effet? C'est un puissant camouflet qui heurte le monde musical et le pousse à réfuter Rousseau en le faisant réfléchir sur lui-même. Si Rousseau avait développé des arguments logiquement cohérents et académiques, ils auraient été plus plausibles, voire irréfutables. Ils auraient pu convaincre le monde musical mais ils n'auraient pas provoqué cette polémique. Ses arguments n'auraient pas pu prêter le flanc à ses détracteurs. Cette "Lettre» en apparence brutale a donc eu pour conséquence de bouleverser les idées établies.

Je suis même tentée de supposer que Rousseau lui-même ait eu l'intention de créer cet effet sans même avoir un élément de théorie. Si tel était le cas, ceux qui ont réagi contre Rousseau se sont laissé prendre à son piège. De toute façon, le fait est que beaucoup de pamphlets contre Rousseau apparaissent successivement après sa "Lettre sur la Musique Françoise». Parmi ces pamphlets j'aimerais vous présenter ici une lettre anonyme qui est, à mon avis, très importante pour appréhender la Querelle des Bouffons du point de vue de l'histoire de l'esthétique musicale.

\section{Une notable lettre anonyme}

Cette lettre est la "Lettre IV»14 insérée dans "Suites des lettres sur la musique françoise. En réponse à celle de Jean-Jacques Rousseaus 15. On peut y lire une préface probablement écrite par Fréron ${ }^{16}$ dont nous en citons une partie.

Elle m'a paru renfermer des idées neuves \& philosophiques, \& je crois qu'elle peut contribuer à augmenter les lumières que la dispute présente nous aura fait acquérir sur la Musique. C'est le seul bien, \& ce n'en est pas un médiocre, que l'Écrit du Génevois nous aura procuré17.

Je suis tout à fait d'accord avec lui. L'un des résultats les plus fructueux de la "Querelle» a consisté à apporter des idées neuves dans le domaine de l'esthétique musicale. En ce sens le rôle de la "Lettre" de Rousseau a été très important parce qu'il a beaucoup 
contribué à lancer des discussions libres en provoquant justement la polémique. L'auteur de la préface, probablement Fréron, prétend qu'il a reçu 12 lettres mais que seule la «Lettre IV» méritait d'être publiée. Elle le fut de façon anonyme. Je cite le paragraphe dans lequel l'auteur anonyme réfute l'opinion de Rousseau selon laquelle la musique italienne peut exprimer tous les caractères qu'elle souhaite grâce à son extrême précision de mesure :

Je ne puis, Monsieur, adopter cette proposition. Je pense que La Musique proprement dite est impuissante à exprimer tous les caractères de paroles imaginables. Voici les raisons sur lesquelles j'appuie mon sentiment. L'organe de l'ouie, par lequel la Musique nous est transmise, est extrêmement borné. L'âme ne peut recevoir d'impressions par cette voie que celles qui naissent uniquement du bruit ou du son. L'art de la Musique, imitateur de la Nature, ne peut donc rappeler à lâme que les objets qui font quelques bruits, ou rendent quelques sons ${ }^{18}$.

L'auteur souligne l'impuissance ou l'imperfection de la musique du point de vue de «l'imitation de la nature»; or il faut se rappeler que la théorie musicale que Rousseau possède reste fondée sur le principe de «l'imitation de la nature". Dans ce sens on peut dire que Rousseau ne s'est pas émancipé du dogme esthétique de l'époque. Bien sûr, à cette époque la plupart des théoriciens approuvent ce principe comme le seul faisant autorité. II est donc d'autant plus notable que l'auteur de la "Lettre IV» fait remarquer l'impossibilité de l'application complète de ce principe à la musique. Partant de cette observation il conclut que la musique ne peut exprimer ni idées ni sentiments.

Je me crois en droit de coclurre(sic) de ce que je viens de dire, que le Compositeur de Musique doit renoncer non-seulement à l'expression des pensées, mais même à celle des sentimens, \& connoissant les bornes de son art, s'y renfermer, \& ne tenter : $1^{\circ}$ que limitation des choses sonores ou bruyantes; $2^{\circ}$ que de faire naître en nous, par le rapport des cordes de l'harmonie à l'arrangement des nerfs, les dispositions propres à nous rendre plus sensibles les situations où le Poète le conduit 19 . 
Du point de vue de «l'imitation de la nature», la capacité de la musique étant limitée, on doit chercher une autre voie musicale pour son expression. Dans son explication, il faut remarquer deux points importants.

Le premier point concerne l'action immédiate de la musique sur le corps humain. Elle agit directement sur les nerfs. Pour expliquer ce fonctionnement de la musique, l'auteur a recours à l'exemple du phénomène acoustique de la résonance entre deux cordes. Selon lui, si l'on fait vibrer une corde, une autre corde accordée convenablement, surtout à l'unisson, commence à vibrer et à rendre du son d'elle-même ${ }^{20}$. II considère que le rapport de l'harmonie et des nerfs est analogue à celui de ces deux cordes ${ }^{21}$. Deuxièmement il faut noter l'utilisation du mot "dispositions" pour indiquer les sensations que la musique suscite. II explique lui-même pourquoi il utilise le mot "dispositions".

l'âme ne pourra recevoir qu'une impression imparfaite : d'où il resultera que ce sera plûtôt une disposition aux passions qu'elle éprouvera, que les passions mêmes22.

II utilise le mot "dispositions" pour souligner l'imprécision des sensations que la musique nous inspire. On ne peut les identifier aux passions ou aux sentiments. Disons que ce sont des sensations purement musicales. Même si quelquefois la force d'un texte semble être en harmonie avec la musique, il faut savoir que cette harmonie est toujours imparfaite. À ce propos il faut aussi citer sa remarquable déclaration : «la musique n'a pas pour but d'exprimer le sens d'un texte".

j'ose avancer qu'il n'y en a pas une (arriette)[...] sur le chant de laquelle on ne pût mettre des paroles d'un caractère différent de celles qui y sont.

II faut donc chercher la cause du plaisir que nous font ces morceaux de Musique ailleurs que dans la véritable expression des paroles qu'on n'y chercheroit en vain23.

Cette indication est très importante parce qu'elle nous rappelle les célèbres phrases de Hanslick qui a été, environ un siècle plus tard, l'un des principaux représentants de la théorie autonome de la musique.

Vous trouverez, par exemple, dans une mélodie dramatique très effective et qui doit exprimer la colère, un seul mouvement 
rapide et passionné. Vous ne trouverez aucune autre expression psychique dans la mélodie elle-même. Les mots qui expriment un mouvement passionné de l'amour, c'est-à-dire, un sentiment complètement opposé à la colère, pourraient être aussi bien interprétés par cette même mélodie24.

En citant Hanslick, j'ai peut- être tort d'omettre des théoriciens comme l'abbé Morlet, Boyé et Chabanon qui ont été des représentants des nouvelles tendances de la théorie autonome de la musique dans le dernier quart du XVIIle siècle en France. Cette "Lettre IV" porte la date du 18 janvier 1753. Nous pouvons donc dire qu'elle est un exemple précurseur de la première période de ces tendances. Cette «Lettre IV» est un court pamphlet de 15 pages. Elle n'est certes pas une théorie complètement développée, pourtant elle contient des remarques très importantes que nous pouvons considérer comme la racine de la théorie autonome de la musique.

\section{Conclusion}

J.-J. Rousseau souligne nécessairement la particularité des styles nationaux parce que dans sa théorie musicale c'est la langue qui détermine le caractère de la musique. Ce qui l'empêche de considérer la musique séparément des paroles, c'est surtout le fait qu'il n'est pas libéré du vieux préjugé de "l'imitation de la nature", considéré d'ailleurs à cette époque comme le principe faisant autorité. II admire tellement le style italien de l'opera buffa qu'il dénie l'existence de la musique française dans sa "Lettre sur la Musique Françoise». Pour interpréter son dernier paragraphe, il faut prendre en considération deux motifs. L'un politique consiste à critiquer l'ancien régime en attaquant l'opéra français. L'autre concerne la rhétorique pour provoquer la polémique.

Parmi les nombreuses discussions causées par sa "Lettre», on peut voir apparaître des idées neuves pour définir la musique. À l'opposé de Rousseau, l'auteur de la "Lettre IV", essaie de considérer la musique en elle-même indépendamment des paroles. II souligne l'imperfection qu'a l'expression musicale non seulement à imiter la nature mais aussi à traduire la force d'un texte. La musique agit directement sur nos nerfs sans intermédiaire de l'expression. Cette explication basée sur le "sensualisme" de l'effet musical validera le concept universel du plaisir musical. L'auteur dit aussi que les sensations que la musique nous donne sont si imparfaites que nous 
ne pouvons les identifier aux passions. Elles sont purement musicales et n'ont pas d'équivalent hors de la musique. De cette tautologie il n'y a qu'un pas jusqu'à la théorie autonome de la musique.

\section{Akiko Koana \\ Tama Art University, Tokyo \\ Koana@tamabi.ac.jp}

1. François Raguenet, Parallèle des Italiens et des Français en ce qui regarde la musique et les opéras (1702), Défense du Parallèle des Italiens et des Français en ce qui regarde la musique et les opéras (1705), Minkoff Reprint Genève 1976.

2. Le Cerf de la Viéville, Jean Laurent, Comparaison de la musique italienne et de la musique française (1704 1706), Minkoff Reprint Genève 1972.

3. François Lesure, (éd), Querelle des Gluckistes et des Piccinnistes, Minkoff Reprint Genève 1984.

4. Voir à ce sujet Koana, Akiko, "Formation de la notion de style national au XVIIIe siècle en France dans le domaine de l'esthétique musicale", à paraître dans les actes du XVe Congrès International d'Esthétique au Japon en 2001.

5. La première fut en 1701. La première reprise fut en 1721et la deuxième reprise en 1733.

6. Denise Launay (éd.), La Querelle des Bouffons, Edition Minkoff, Genève, 1973.

7. Selon le numérotage de Launay, la première partie consiste en des documents de 1 à 4 , la seconde partie de 5 à 32 et la troisième partie de 33 à 61 .

8. Denise Launay, Tome I, I 1-54, Lettre de M.Grimm sur Omphale, Tragédie Lyrique, reprise par l'Académie Royale de Musique le 14 janvier 1752.

9. Ibid., Tome I, IV 87-117, Lettre à M.Grimm, Au sujet des Remarques ajoutées à sa Lettre sur Omphale.

10. Sur ce point, voir Masson(1944).

11. Boyer (1945) appelle notre attention sur l'usage particulier à Rousseau du mot "remplissage». Selon Boyer, cet usage indique le mépris ou l'incompréhension de Rousseau pour l'harmonie.

12. Denise Launay, Tome I, XXXIII 763-764 (p. 91-92).

13. d'Alembert, Liberté de la musique, Launay, Tome III, LXI 2275 (p.455).

14. Denise Launay, Anonyme, Tome II, XLI $1025 \mathrm{ff}$.

15. Denise Launay, Tome II, XLI 1007ff.

16. Elie-Catherine Fréron (1718-1776) est critique et journaliste contre les Encyclopédistes. La lettre en question a été à l'origine publiée dans ses "Lettres sur quelques écrits de ce temps", 1749-1754, Slatkine reprint, $2^{\theta}$ vol. Genève, 1922. Tome II, p. 481 f. Elle porte la date de "Paris 18 janvier 1753".

17. Denise Launay, Tome II, XLI 1025 (p. 21).

18. Denise Launay, Tome II, XLI 1026 (p. 22). 
19. Ibid., Tome II, XLI 1030 (p. 26).

20. Ibid., Tome II, XLI 1027 (p. 23).

21. À ce propos, il faut mentionner aussi qu'il parle de "la sensibilité des animaux" et de "la guérison de la Tarentule» pour expliquer ce mécanisme de l'effet de la musique sur l'âme. (lbid., Tome II, XLI 1028 (p.24)).

22. Ibid., Tome II, XLI 1028 (p. 24).

23. Denise Launay, Tome II, XLI 1035-1036 (p. 31-32).

24. Ma traduction. "Man wird z.B. in einer sehr wirksamen dramatischen Melodie, welche Zorn auszudrücken hat, an und für sich keinen weiteren psychischen Ausdruck finden, als den einer raschen, leidenschaftlichen Bewegung. Worte einer leidenschaftlich bewegten Liebe, also das gerade Gegenteil, werden vielleicht gleich richtig durch dieselbe Melodie interpretiert sein."

Eduard Hanslick, Vom Musikalisch=Schönen (1854), Breitkopf \& Härtel, Wiesbaden, 1989, p. 37

25. II faut parler aussi de son opposition au "sensualisme».

\section{Bibliographie concernant la Querelle des Bouffons}

Anonyme (1753), Lettre à Madame Favart, Cologne : chez Pierre Marteau.

Blainville, Cherles Henri de (1754), L'Esprit de l'art ou Réflexions sur la musique et ses defférentes Parties, Genève, Minkoff Reprint, 1974.

Borrel, Eugine (1963), "La Querelle des Bouffons", dans Encyclopédie de la Pléiade : Histoire de la musique II, Paris, p.191-206.

Bottacin, Annalisa (1991), Jacques Cazotte e la "Querelle des Bouffons", Este, Libreria Editrice Zielo.

Boyer, Noël (1945), La Guerre des bouffons et la musique française 1752-1754, Paris.

Cucuel, George (1914), Les créateurs de l'opéra-comique française, Paris.

Di Profio, Alessandro (1999), L'opera buffa à Paris : le cas du Théâtre de Monsieur et du Théâtre Feydeau : 1789-1792, Tours, ToursUniv. Fr. Rablais.

Freron, Elie-Catherine (1753-1754), Lettres sur quelques écrits de ce temps, Tome II, Genève, Slatkine, 1966.

Funayama, Nobuko (1979), «Étude sur la Querelle des Bouffons(I)» Le bulletin de l'Université Uenogakuen au $75^{\circ}$ anniversaire de la fondation, p. 127-140 (en japonais). 
Funayama, Nobuko (1989), «Étude sur la Querelle des Bouffons(II)» Le bulletin de l'Université Uenogakuen au $85^{\circ}$ anniversaire de la fondation, p. 252-271 (en japonais).

Goudar, Ange (1777), Le Brigandage de la musique italienne, Genève, Minkoff Reprint, 1972.

La Laurencie, Lionel de (1912), "La grande saison italienne de 1752 : les Bouffons", dans Société Internetionnale de Musicologie.

La Querelle des Bouffons, 3 vols, (1973) éd par Denise Launay, Genève, Minkoff.

Massin, Marienne (1996), "Jean-Jacques Rousseau ou La transparence musicale contre le pouvoir des intermédiaires", dans Rousseau, Jean-Jacques. Lettre d'un symphoniste de l'Académie royale de musique à ses camarades de l'orchestre, Rumeur des Âges.

Masson, Paul-Marie (1944), "La "Lettre sur Omphale" " dans Revue de Musicologie XXIII, p.1-19.

Pekack, Jolanta T. (1999), Conservative tradition in pre-revolutionary France : Parisian salon women, New York, Peter Lang Publishing.

Pougin, Arthur (1880), Biographie universelle des musiciens et bibliographie générale de la musique par F.-J. Fétis, tome II Supplément et complément, Paris / Bruxelles(1963).

Richebourg, E. Louisette (1937), Contribution à l'histoire de la "Querelle des Bouffons", Paris.

Russo, Paolo (1997), La parola $\theta$ il gesto: studi sull'opera francese nel Settecento, Lucca, Libreria musicale italiana.

Travenol, Louis $(1757,1762)$, Histoire du Théâtre de l'Académie royale de musique en France, depuis son établissement jusqu'à présent, Paris. 